\title{
MicroRNA-23b Inhibits Enterovirus 71 Replication through Downregulation of EV71 VPI Protein
}

\author{
Bai-ping Wen \\ Hong-jian Dai \\ Kunming Children's Hospital, Kunming, China
}

\section{Key Words}

Enterovirus $71 \cdot \mathrm{miR}-23 \mathrm{~b} \cdot$ MicroRNAs $\cdot$ EV71 VPI protein

\begin{abstract}
Enterovirus 71 (EV71) is one of the causative pathogens of hand-foot-and-mouth disease and effective antiviral agents and vaccines against this virus have, to date, not been available. MicroRNAs (miRNAs) are a recently discovered class of RNAs with the function of post-transcriptional gene expression regulation. It has been demonstrated that miRNAs play important roles in the complicated interaction network between virus and host, while few studies have explored the role of miRNAs in EV71 infection. A recent study showed that hsa-miR-23b was downregulated significantly in cell-infected viruses. To address this issue, biological software miRanda was first used to predict possible target sites of miR-23b at EV71 gene sequence, then to confirm it by luciferase assay. miR-23b mimics were transfected to verify its effects on infection of EV71. These results suggest that miR-23b and upregulation of miR-23b inhibited the replication of EV71 by targeting at EV71 3'UTR conserved sequence. Taken together, miR-23b could inhibit EV71 replication through downregulation of EV71 VPI protein. These results may enhance our understanding on the prevention and treatment of hand-foot-and-mouth disease caused by EV71 infection.
\end{abstract}

Copyright $\odot 2013$ S. Karger AG, Basel
() 2013 S. Karger AG, Basel

0300-5526/13/0563-0195\$38.00/0

\section{Yu Zhuang Ru Sheng}

\section{Introduction}

Enterovirus 71 (EV71) was first isolated in 1969 from stool specimens of infants with central nervous system diseases in California [1], which is now identified as one of the causative pathogens to infant hand-foot-andmouth disease (HFMD). Since being first reported in 1974, EV71 has induced several epidemic outbreaks in the world [1]. In China, it was found that subtype C4 was the main type of EV71 in the 1998 epidemic [2]. According to the existing data, HFMD caused by EV71 was of larger severe infection proportion and higher mortality compared with other enteroviruses. Acute EV71 infection also causes serious nervous system disease, or even fatal disease deterioration [3]. EV71 belongs to a small RNA virus family with an infectious positive singlestrand RNA [4]. Up to now, no specific vaccines, antiviral drugs or therapies against EV71 infection are available. The underlying mechanisms through which EV71 infection induced serious cerebral and pulmonary complications and even death are not clear. Therefore, further investigation on the pathogenesis of EV71 infection has kindled great interest of scientists in the field of medicine and biology.

B. Wen and H. Dai contributed equally to this work.

\section{KARGER}

E-Mail karger@karger.com www.karger.com/int
E-Mail ketixiezuo@126.com 
MicroRNAs (miRNAs) are short noncoding ribonucleic acid molecules ( 22 nucleotides) which function to modulate the activity of specific mRNA target and play a critical role in a wide range of physiologic and pathologic processes, such as cell growth, proliferation, differentiation and apoptosis, by post-transcriptional regulation of target gene expression [5]. When miRNA sequences are completely complementary to the $3^{\prime}$-untranslated region (UTR) of target mRNA, target mRNA will be cut. Otherwise, translation of target protein would be inhibited without affecting its mRNA stability. Most of the currently known miRNAs in human cells are incompletely complementary to the target gene sequences and thus play negative regulatory roles on target genes by inhibiting their translation. Recent studies have revealed that miRNAs expressions are frequently dysregulated in the development of a variety of cancers and demonstrated that miRNAs are a promising class of biomarker candidates for clinical cancer diagnosis $[5,6]$.

As an important kind of post-transcriptional gene expression regulatory molecule, miRNAs act as key effector molecules in the complicated interaction network between virus and host [6]. Both virus and host could encode miRNAs. Virus miRNAs resist to human's antiviral immune defense system by changing various gene expressions to control cell growth and development. Cellencoded miRNAs could directly affect the virus replication cycle. Therefore, studies on miRNA level could contribute to further understanding of the mechanisms of the interaction between virus and the host cells, and provide scientific information for discovery of novel antiviral agents and strategies. Recently, Kelly et al. [7, 8] showed that miRNAs interfered with viruses in the whole life cycle of enterovirus and Coxsackie A21 through degradation of viral RNA genome, inhibition of mRNA translation and interference with virus capsid. However, studies are needed to explore the miRNA-mRNA interaction between EV71 virus and host cells, and to elucidate the role of EV71 in the pathogenesis. By cloning and sequencing analysis of HPV16-positive CaSki cells, Wang et al. [9] found that the expression level of miR-23b in the cells decreased significantly. Similarly, Au Yeung et al. [10] showed that miR-23b was downregulated in HPV-associated cervical cancer. Considering that related studies of miR-23b and EV71 have not been reported yet, biological software miRanda was used to predict the target genes and the results showed that miR-23b targeted the conservative sequence of EV71 3'UTR. Therefore, it can be inferred that miR-23b may play an important regulatory role in EV71. In the present study, transfection with miR- 23b mimics were performed, followed by infection with EV71 to explore the regulating function of miR-23b towards EV71. Further experiments were also carried out to verify the target site of miR-23b at EV71.

\section{Materials and Methods}

\section{Cell Culture}

Vero cells (ATCC, CCL-81) were cultured in Dulbecco's modified Eagle's medium (Invitrogen) supplemented with 5\% heatedinactivated fetal bovine serum (HyClone) and penicillin-streptomycin at $37^{\circ}$ in a humidified atmosphere containing $5 \% \mathrm{CO}_{2}$.

\section{miRNA Mimics Transfection and EV71 Infection}

Vero cells were inoculated in a 24-well plate and incubated for $24 \mathrm{~h}$. Transfection was performed following the manufacturer's instructions of Lipofectamine 2000 (Invitrogen) when the cell confluence reached about $50 \%$. The final concentration of hsamiR-23b mimics or mimic Negative Control (RiboBio, China) was $50 \mathrm{nM} /$ well. After $24 \mathrm{~h}$, Vero cells were infected with $100 \mu \mathrm{l}$ $50 \%$ tissue culture infective dose $\left(\mathrm{TCID}_{50}\right)$ of the EV71 strain EV71/Fuyang.Anhui.P.R.C/17.08/1 (GenBank accession No. EU703812). Unbound viruses were washed away $2 \mathrm{~h}$ after infection and cells were then cultured in fresh medium containing $10 \%$ fetal bovine serum. The cells were collected for subsequent analysis after $48 \mathrm{~h}$.

\section{Real-Time RT-PCR}

The cDNA was prepared from total RNA using AMV reverse transcriptase $(\mathrm{TaKaRa})$. Briefly, $20 \mu \mathrm{l}$ reaction mixture was incubated for $15 \mathrm{~min}$ at $16^{\circ}, 30 \mathrm{~min}$ at $42^{\circ}$ and $5 \mathrm{~min}$ at $85^{\circ}$ in succession, and then maintained at $4^{\circ}$. Quantitative real-time PCR was performed using the Applied Biosystems 7500 Sequence Detection system (Applied Biosystems), with $1 \mu \mathrm{l}$ of RT-PCR product, $10 \mu \mathrm{l}$ of Premix Ex Taq (TaKaRa), and $1 \mu \mathrm{l}$ of SYBR green (Invitrogen) contained in $20 \mu \mathrm{l}$ of reaction mixture. The reaction was performed at one cycle of $95^{\circ}$ for $10 \mathrm{~min}$, followed by 40 cycles of $95^{\circ}$ for $15 \mathrm{~s}$ and $60^{\circ}$ for $1 \mathrm{~min}$ in a 96 -well plate. Three independent experiments were conducted for each sample. Threshold cycle value (CT) data were determined using default threshold settings, and the mean CT was calculated from the duplicate PCRs. The expression levels of miRNAs were measured by CT and normalized to human small nuclear RNA U6 using $2^{-\Delta \Delta C T}$ value [11]. Primers for detection of mature hsa-miR-23b were obtained from Ambion Co.

\section{EV71 Virus Titer $\left(\right.$ TCID $\left._{50}\right)$ Assay}

Virus titers were determined by measuring $\mathrm{TCID}_{50}$ as previously described [12].

\section{EV71 RNA Assay}

Viral RNA was extracted from the culture medium of infected cells using the QIAamp Viral RNA Mini Kit (Qiagen). Real-time RT PCR for the detection of the $5^{\prime} \mathrm{UTR}$ of EV71 was performed as described by Nijhuis et al. [13]. The real-time PCR amplification was cloned into pMD 18-T vector (TaKaRa) and then the plasmid was used as a standard, using the standard curve to quantify the virus. 
EV71 VPl Protein Assay

Total cellular proteins were extracted by incubating cells in lysis buffer $(20 \mathrm{~mm}$ Tris-HCl, pH 7.4, 1\% Nonidet P-40, $137 \mathrm{~mm}$ $\mathrm{NaCl}, 50 \mathrm{~mm}$ EDTA, protease inhibitor mixture and $1 \mathrm{~mm}$ phenylmethylsulfonyl fluoride). After centrifugation at 14,000 rpm for 10 $\min$ at $4^{\circ}$, supernatants were transferred and mixed with an equal amount of protein sample buffer (120 mM Tris-HCl, pH 8.0, 20\% glycerol, $4 \%$ SDS, $2.5 \% \beta$-mercaptoethanol and $0.05 \%$ bromophenol blue). Lysates were separated by SDS-PAGE and transferred to nitrocellulose membrane (Millipore) that had been blocked with $5 \%$ BSA in TBST buffer for $1 \mathrm{~h}$ at room temperature. The membrane was then incubated overnight at $4^{\circ}$ with anti-enterovirus VP1 monoclonal antibody (LifeSpan BioSciences) diluted in the blocking buffer. After hybridization with primary antibody, the membrane was washed three times with TBST before hybridizing with anti-goat HRP secondary antibody (Sigma) diluted in TBST, according to the primary antibody used. The blots were then washed three times with TBST and detected by chemiluminescence (Santa Cruz Biotechnology).

\section{Plasmid Constructions}

The full-length EV71 3'UTR was amplified from cDNA using forward primer $\mathrm{F}\left(5^{\prime}\right.$-CCGCTCGAG AGGTTATACACACCTCAACC- $\left.3^{\prime}\right)$ and the reverse primer R ( $5^{\prime}$-ATAAGAATGCGGCCGC GCTATTCTGGTTATAACAAATT-3'). The other two primers (mutF + mutR) were used to generate mutant-type EV71 $3^{\prime} \mathrm{UTR}$ in which the four mutated nucleotides were underlined within the seed region of the miR-23b binding site by a PCR-based mutagenesis method. Both PCR fragments were cloned into psiCHECK-2 vector (Promega). The primer sequences were mutF (5'-CCGCTCGAG AGGTTATACACACCTCAACCCCACCAGAAATCTGG TCGTGCGTATCACTG- $\left.3^{\prime}\right)$ and mutR ( $5^{\prime}$-ATAAGAATGCGGCCGC GCTATTCTGGTTATAACAAATTTA CCCC CACCAGTGATACGCACGA-3'), respectively.

\section{Luciferase Assay}

Reporter vector psiCHECK-2 carrying the 3 'UTR sequences of EV71 were assayed for luciferase expression using the Dual Glo Luciferase Assay System (Promega) according to the manufacturer's instructions. The relative expression of target-specific Renilla luciferase was determined by comparing the normalized levels with the control firefly luciferase for each sample transfected a minimum of three replicates \pm standard error and was representative of at least two independent experiments.

\section{Results}

\section{EV71 Infection Downregulates the Expression of $\operatorname{miR}-23 b$}

Fluorescence quantitative PCR detection showed a significant decrease in miR-23b expression in Vero cells infected with EV71 alone (fig. 1), indicating that EV71 could inhibit the expression of miR-23b. In Vero cells transfected with miR-23b mimics + EV71, miR-23b expression was lower than that without EV71 infection, but was still a dramatic rise (fig. 1).

Inhibition of Enterovirus 71 Replication by $\mathrm{miR}-23 \mathrm{~b}$

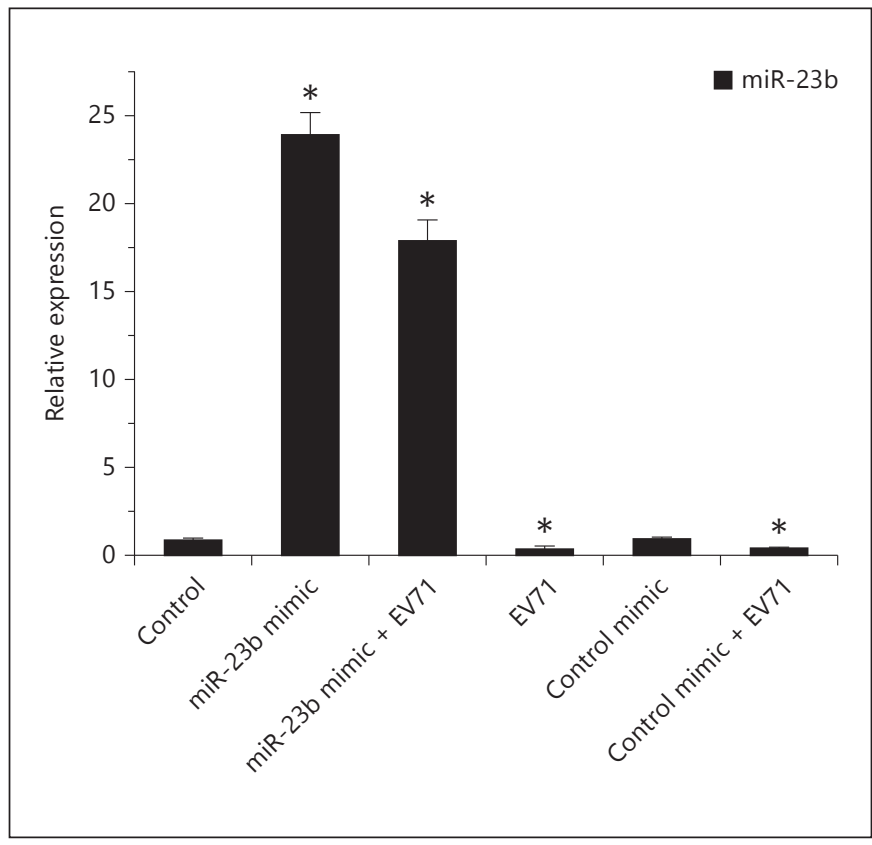

Fig. 1. Real-time PCR of miR-23b expression. Control = Vero cells alone; miR-23b mimic $=$ Vero cells transfected with miR-23b mimics; miR-23b mimic + EV71 = Vero cells infected with EV71 and transfected with miR-23b mimics; EV71 = Vero cells infected with EV71 alone; Control mimic = Vero cells transfected with mimic Negative Control; Control mimic + EV71 = Vero cells infected with EV71 and transfected with mimic Negative Control. Each bar represents mean \pm SD from three samples (* $\mathrm{p}<0.01$ vs. Control). Human small nuclear RNA U6 was used as a loading control.

\section{miR-23b Inhibits Replication of EV71}

A cytopathic effect occurred $72 \mathrm{~h}$ after Vero cells were transfected with miRNA mimics and inoculated with EV71, and cell death appeared. Supernatant was collected and detected by EV71 virus titer $\left(\mathrm{TCID}_{50}\right)$ assay. The results showed that after transfection with miRNA mimics, EV71 virus titer $\left(\right.$ TCID $\left._{50}, \log _{10} / 50 \mu \mathrm{l}\right)$ decreased from 5.86 to 4.25 . That is to say, in every $0.05 \mathrm{ml}$ of virus solution there are $10^{5.86} \mathrm{TCID}_{50}$ viruses dropping to $10^{4.25} \mathrm{TCID}_{50}$ viruses, which is only $2.45 \%$ of the former (fig. 2).

However, after detection of EV71 RNA content, results of fluorescence quantitative PCR showed no obvious changes or statistical significance among the groups, which indicated that miR-23b did not affect the EV71 RNA level. Nevertheless, after detection of supernatant EV71 VPl protein by Western blot, it was found that the EV71 VPl protein expression level of the miR-23b mimics transfected group decreased (fig. 3). 


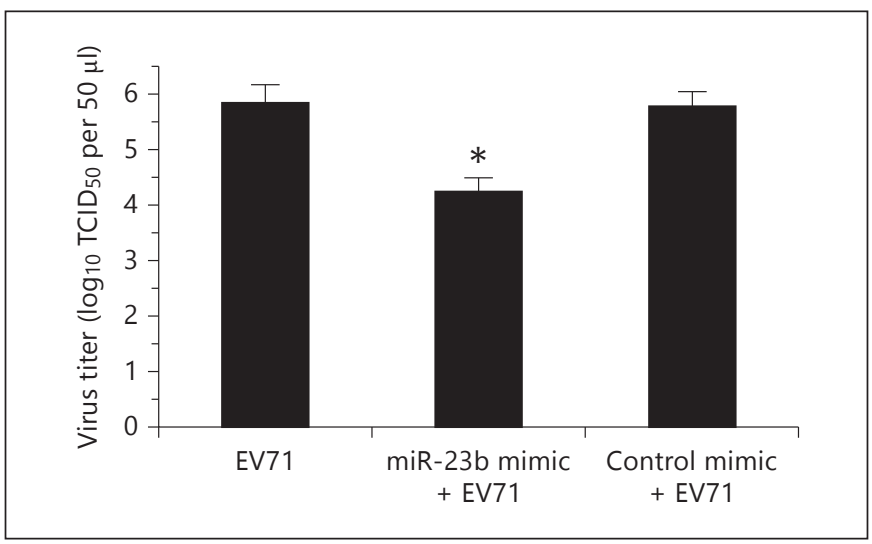

Fig. 2. EV71 virus titer $\left(\mathrm{TCID}_{50}\right)$ assay. Each bar represents mean \pm SD of three samples $(* \mathrm{p}<0.01)$.

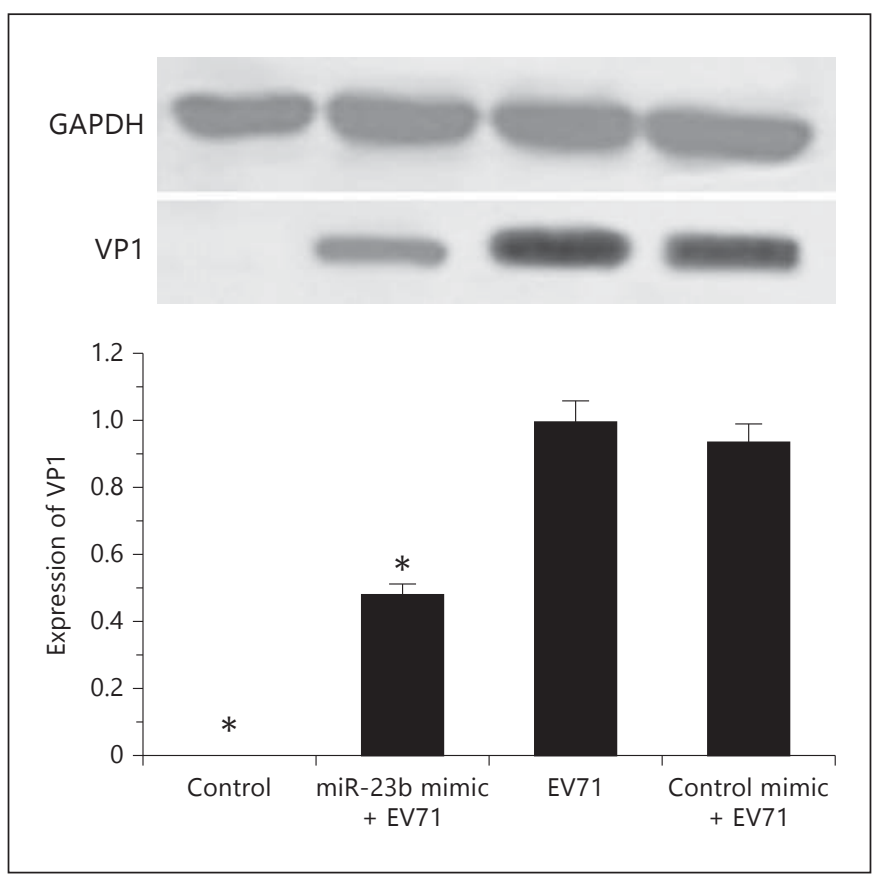

Fig. 3. EV71 VPl protein detected by Western blot. Control = Vero cells alone; miR-23b mimic + EV71 = Vero cells infected with EV71 and transfected with miR-23b mimics; EV71 = Vero cells infected with EV71 alone; Control mimic + EV71 = Vero cells infected with EV71 and transfected with mimic Negative Control. Each bar represents mean \pm SD of three samples (* $p<0.01$ vs. EV71-infected group).

\section{Prediction of miR-23b Target Gene}

The previous results have showed that miR-23b exerted no effect on EV71 RNA content. However, an increase in its expression effectively suppressed the expression of EV71 VPl protein, leading to a decrease in EV71 virus titer
$\left(\mathrm{TCID}_{50}\right)$. These results imply that an increase in expression of miR-23b could inhibit the replication of EV71. Therefore, biological software miRanda was used to predict the targeting sites of miR-23b in the conservative sequence of EV71 3'UTR. It was found that miR-23b may have targeting sites in the conservative sequence of EV71 3'UTR, which was incompletely matched with EV71 $3^{\prime}$ UTR with 7 consecutive nucleic acids matched (fig. 4a). Therefore, it could be inferred that miR-23b is incompletely complementary to EV71 $3^{\prime}$ UTR mRNA to play a negative regulatory role in inhibition of EV71 replication.

\section{miR-23b Regulates Expression of EV71 Gene}

EV71 3'UTR was cloned into the dual luciferase reporter vector psiCHECK-2, and target sites of miR-23b were mutated. Cloned EV713'UTR vector, mutantEV713'UTR vector and miR-23b mimics were respectively transfected into the cells, and the change in expression of luciferase was analyzed. Taking cells transfected with reporter vector as a control, the luciferase expression level of the cells transfected with cloned EV71 3'UTR vector significantly decreased about $60 \%$ (fig. 4b), while luciferase expression of the cells transfected with mutant EV71 $3^{\prime} \mathrm{UTR}$ vector showed no apparent change. Taken together, these results validate that miR-23b could target EV71 3'UTR.

\section{Discussion}

In the past years, morbidity and mortality due to EV71 infection have tended to increase, whereas the underlying pathogenic mechanisms have remained elusive, and effective vaccines or therapies are therefore urgently needed. miRNAs play an important regulatory role in the cells by targeting $\mathrm{mRNAs}$ to regulate different gene expression [14]. Studies have showed that miRNAs encoded by viruses and host play important roles in complicated signaling pathways of the virus-host interaction network [1416]. In order to investigate the effects of miR-23b on EV71, a biological software miRanda was used to predict the possible target sites of miR-23b in the conservative sequence of EV71 $3^{\prime}$ UTR. It was found that miR-23b was incompletely complementary to the conservative sequence of EV71 3'UTR through 7 consecutive nucleic acids. Therefore, it could be speculated that miR-23b regulates the gene expression of EV71 and could contribute to the treatment of EV71 infection.

In Vero cells, transfection with miR-23b mimics was performed, followed by infection with EV71. The detection of miR-23b expression level showed that expression 


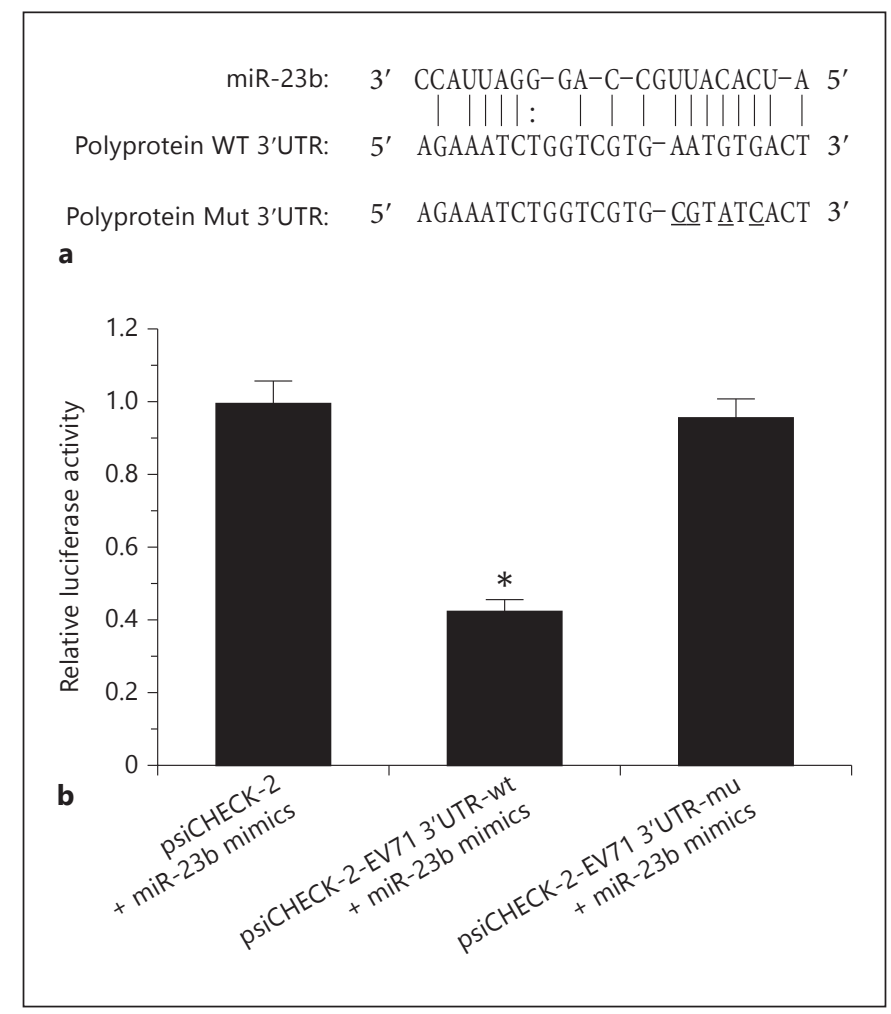

Fig. 4. Prediction of miR-23b target genes and the luciferase expression level. a miR-23b target sites in the conservative sequence of EV71 3'UTR. b Luciferase expression level of cells transfected with cloned EV71 3'UTR vector or mutant EV71 3'UTR vector and $\mathrm{miR}-23 \mathrm{~b}$ mimics. Each bar represents mean \pm SD of three samples $\left({ }^{*} \mathrm{p}<0.01\right)$.

of miR-23b was inhibited by EV71 infection, but still increased dramatically after transfected with miR-23b mimics, which was consistent with the results on HPV16 reported by Wang et al. [9] and Au Yeung et al. [10]. Similarly, Cui et al. [17] utilized high-throughput sequencing technology to explore the change in miRNAs in EV71infected Hep2 cells. The results revealed that EV71 infection was closely linked to miRNA, without involvement of miR-23b. Recently, Cui et al. [18] used TaqMan lowdensity array to analyze the miRNA levels in the serum of HFMD patients infected with EV71, and they showed that the expression level of some miRNAs still had significant changes, by comparing with healthy patients, also without the involvement of miR-23b as well. Comparing the two studies conducted by Cui et al. [18], rare superposition of significantly changed miRNAs was found and miR-545 was opposite. Meanwhile, it was found that after transfection with miR-23b mimics, although the EV71 RNA level remained, an increase in the expression of miR-23b inhib- ited the EV71 VPl protein expression and led to a decrease in EV71 virus titer $\left(\mathrm{TCID}_{50}\right)$, suggesting that an increase in the expression of miR-23b inhibited replication of EV71. Previous studies have also provided supportive proof of this conclusion. For instance, the results from $\mathrm{Du}$ et al. [19] showed that vector-delivered miRNAs targeting the 3D genes efficiently inhibited the FMDV replication in vitro. Ahluwalia et al. [20] found that human cellular miRNA hsa-miR-29a interfered with the expression of viral Nef protein and HIV-1 replication. Sung and Rice [21] demonstrated that miR-198 suppressed HIV-1 gene expression and replication in monocytes. Song et al. [22] found that the cellular miRNAs inhibited the replication of the H1N1 influenza A virus in infected cells. Othumpangat et al. [23] confirmed that miRNA-221 modulated the RSV replication in human bronchial epithelium by targeting the NGF expression.

Furthermore, results from dual luciferase assay validated that miR-23b targeted EV71 incompletely complementary to EV71 3'UTR and thus inhibited protein translation of EV71 without affecting the mRNA level. Tan et al. [24] had shown that synthetic 19-mer siRNAs and plasmid-borne short hairpin RNAs (shRNAs) targeted at the conserved 3Dpol region and inhibited the EV71 infection in suckling mice. Wu et al. [25] adopted RNA interference (RNAi) as an antiviral agent to inhibit EV71 replication in rhabdomyosarcoma (RD) cells. The results suggested that 3 small interfering RNAs (siRNAs) targeting extremely conserved regions of multiple EV71 strains effectively blocked the replication of EV71 strain Shzh98. Although the mechanisms of siRNA and miRNA have much in common, studies on siRNA and EV71 are more sufficient than miRNA and EV71. Except for the studies of Cui et al. [17, 18], Ho et al. [26] demonstrated that enterovirus-induced miR-141 contributed to shutoff of host protein translation by targeting the translation initiation factor eIF4E.

Nowadays, EV71 infection is still one of the most common global-threatening viruses with multiple manifestations depending on the time, region, individual and virus types [27]. The gene mutation mechanisms of EV71 virus subtypes are still ambiguous, and the effects of the difference in genome sequence on the function of the virus remain elusive. In the present study, we found that miR-23b could inhibit EV71 replication through downregulation of EV71 VPl protein. These results may enhance our understanding on the prevention and treatment of HFMD caused by EV71 infection. Moreover, the results from the present study many contribute to the better understanding of host-virus interaction mechanisms. 


\section{References}

$>1$ Schmidt NJ, Lennette EH, Ho HH: An apparently new enterovirus isolated from patients with disease of the central nervous system. J Infect Dis 1974;129:304-309.

-2 Zhang Y, Tan XJ, Wang HY, Yan DM, Zhu SL, Wang DY, Ji F, Wang XJ, Gao YJ, Chen L, An HQ, Li DX, Wang SW, Xu AQ, Wang ZJ, $\mathrm{Xu}$ WB: An outbreak of hand, foot, and mouth disease associated with subgenotype c4 of human enterovirus 71 in Shandong, China. J Clin Virol 2009;44:262-267.

$>3$ Huang CC, Liu CC, Chang YC, Chen CY, Wang ST, Yeh TF: Neurologic complications in children with enterovirus 71 infection. N Engl J Med 1999;341:936-942.

$\checkmark 4$ Phuektes P, Chua BH, Sanders S, Bek EJ, Kok CC, McMinn PC: Mapping genetic determinants of the cell-culture growth phenotype of enterovirus 71. J Gen Virol 2011;92:13801390.

$\checkmark 5$ Carrington JC, Ambros V: Role of microRNAs in plant and animal development. Science 2003;301:336-338.

6 Kurzyńska-Kokorniak A, Jackowiak P, Figlerowicz M: Human- and virus-encoded microRNAs as potential targets of antiviral therapy. Mini Rev Med Chem 2009;9:927-937.

7 Kelly EJ, Hadac EM, Greiner S, Russell SJ: Engineering microRNA responsiveness to decrease virus pathogenicity. Nat Med 2008; 14: 1278-1283.

8 Kelly EJ, Hadac EM, Cullen BR, Russell SJ: MicroRNA antagonism of the picornaviral life cycle: alternative mechanisms of interference. PLoS Pathog 2010;6:e1000820.

$\checkmark 9$ Wang X, Tang S, Le SY, Lu R, Rader JS, Meyers C, Zheng ZM: Aberrant expression of oncogenic and tumor-suppressive microRNAs in cervical cancer is required for cancer cell growth. PLoS One 2008;3:e2557.

10 Au Yeung CL, Tsang TY, Yau PL, Kwok TT: Human papillomavirus type 16 E6 induces cervical cancer cell migration through the p $53 / \mathrm{microRNA}-23 \mathrm{~b} /$ urokinase-type plasminogen activator pathway. Oncogene 2011;30:2401-2410.
11 Schmittgen TD, Livak KJ: Analyzing realtime PCR data by the comparative $\mathrm{C}(\mathrm{T})$ method. Nat Protoc 2008;3:1101-1108.

-12 Chua BH, Phuektes P, Sanders SA, Nicholls PK, McMinn PC: The molecular basis of mouse adaptation by human enterovirus 71 . J Gen Virol 2008;89:1622-1632.

13 Nijhuis M, van Maarseveen N, Schuurman R, Verkuijlen S, de Vos M, Hendriksen K, van Loon AM: Rapid and sensitive routine detection of all members of the genus enterovirus in different clinical specimens by real-time PCR. J Clin Microbiol 2002;40:3666-3670.

14 Lecellier CH, Dunoyer P, Arar K, LehmannChe J, Eyquem S, Himber C, Saïb A, Voinnet O: A cellular microRNA mediates antiviral defense in human cells. Science 2005;308: 557-560.

15 Gottwein E, Cullen BR: Viral and cellular microRNAs as determinants of viral pathogenesis and immunity. Cell Host Microbe 2008;3: 375-387.

16 Grey F, Tirabassi R, Meyers H, Wu G, McWeeney S, Hook L, Nelson JA: A viral microRNA down-regulates multiple cell cycle genes through MRNA 5'UTRs. PLoS Pathog 2010;6:e1000967.

17 Cui L, Guo X, Qi Y, Qi X, Ge Y, Shi Z, Wu T, Shan J, Shan Y, Zhu Z, Wang H: Identification of microRNAs involved in the host response to enterovirus 71 infection by a deep sequencing approach. J Biomed Biotechnol 2010; 2010:425939.

18 Cui L, Qi Y, Li H, Ge Y, Zhao K, Qi X, Guo X, Shi Z, Zhou M, Zhu B, Guo Y, Li J, Stratton CW, Tang YW, Wang H: Serum microRNA expression profile distinguishes enterovirus 71 and coxsackievirus 16 infections in patients with hand-foot-and-mouth disease. PLoS One 2011;6:e27071.
19 Du J, Gao S, Luo J, Zhang G, Cong G, Shao $\mathrm{J}$, Lin T, Cai X, Chang H: Effective inhibition of foot-and-mouth disease virus (FMDV) replication in vitro by vector-delivered $\mathrm{mi}$ croRNAs targeting the $3 \mathrm{~d}$ gene. Virol J 2011; 8:292.

20 Ahluwalia JK, Khan SZ, Soni K, Rawat P, Gupta A, Hariharan M, Scaria V, Lalwani M, Pillai B, Mitra D, Brahmachari SK: Human cellular microRNA hsa-miR-29a interferes with viral Nef protein expression and HIV-1 replication. Retrovirology 2008;5:117.

21 Sung TL, Rice AP: miR-198 inhibits HIV-1 gene expression and replication in monocytes and its mechanism of action appears to involve repression of cyclin T1. PLoS Pathog 2009; 5:e1000263.

-22 Song L, Liu H, Gao S, Jiang W, Huang W: Cellular microRNAs inhibit replication of the $\mathrm{H} 1 \mathrm{~N} 1$ influenza A virus in infected cells. J Virol 2010;84:8849-8860.

23 Othumpangat S, Walton C, Piedimonte G: MicroRNA-221 modulates RSV replication in human bronchial epithelium by targeting NGF expression. PLoS One 2012;7:e30030.

24 Tan EL, Tan TM, Tak Kwong Chow V, Poh CL: Inhibition of enterovirus 71 in virus-infected mice by RNA interference. Mol Ther 2007; 15:1931-1938.

25 Wu Z, Yang F, Zhao R, Zhao L, Guo D, Jin Q: Identification of small interfering RNAS which inhibit the replication of several enterovirus 71 strains in china. J Virol Methods 2009;159:233-238.

26 Ho BC, Yu SL, Chen JJ, Chang SY, Yan BS, Hong QS, Singh S, Kao CL, Chen HY, Su KY, Li KC, Cheng CL, Cheng HW, Lee JY, Lee CN, Yang PC: Enterovirus-induced miR-141 contributes to shutoff of host protein translation by targeting the translation initiation factor eIF4E. Cell Host Microbe 2011;9:58-69.

-27 Liu J, Yang Y, Xu Y, Ma C, Qin C, Zhang L: Lycorine reduces mortality of human enterovirus 71 -infected mice by inhibiting virus replication. Virol J 2011;8:483. 